\title{
La terre sous les pieds. Entretien sur l'installation « Fosse commune » et la question des disparus de la guerre civile espagnole
}

The earth beneath the feet. Interview on the installation "Communal grave" and the question of the missing people of the Spanish Civil War

La tierra bajo nuestros pies. Entrevista con Tomás Ruiz-Rivas sobre la instalación "Fosa común" y la cuestión de los desaparecidos durante la guerra civil española

\section{Antonia García Castro et Tomás Ruiz-Rivas}

\section{(2) OpenEdition}

\section{Journals}

Édition électronique

URL : http://journals.openedition.org/conflits/16763

DOI : $10.4000 /$ conflits. 16763

ISSN : $1777-5345$

Éditeur :

CCLS - Centre d'études sur les conflits lilberté et sécurité, L'Harmattan

Édition imprimée

Date de publication : 30 octobre 2008

Pagination : 153-166

ISBN : 978-2-296-06624-3

ISSN : 1157-996X

Référence électronique

Antonia García Castro et Tomás Ruiz-Rivas, «La terre sous les pieds. Entretien sur l'installation « Fosse commune » et la question des disparus de la guerre civile espagnole», Cultures \& Conflits [En ligne], 71 | automne 2008, mis en ligne le 05 février 2009, consulté le 30 mars 2021. URL : http:// journals.openedition.org/conflits/16763; DOI : https://doi.org/10.4000/conflits. 16763 


\title{
La terre sous les pieds. Entretien sur l'installation " Fosse commune" et la question des disparus de la guerre civile espagnole
}

\author{
Antonia GARCIA CASTRO
}

Antonia Garcia Castro est docteur en sociologie et rédactrice associée de la revue Cultures \& Conflits.

\section{Tomás RUIZ-RIVAS}

Tomás Ruiz-Rivas (Madrid, 1962) est fondateur et co-directeur de l'Ojo Atómico. Antimuseo de Arte Contemporéaneo (Oeil atomique. Antimusée d'art contemporain). Il travaille sur les systèmes alternatifs d'art et la formation des publics. Depuis l'année 2000, il travaille également en tant $q u$ 'artiste du visuel, sous le nom de Tom Lavin. Son ouvre est axée sur les questions de l'identité et de la mémoire historique espagnoles.

«Cependant, cela ne fait pas de doute que la société a besoin de constructions symboliques, et plus encore quand elle affronte des faits si difficiles à assumer par la raison. Et c'est de cela dont nous allons parler aujourd'hui, demain et après-demain. Du rôle que joue ou peut jouer l'art dans un débat plus large sur les disparus, de la possibilité de la représentation de l'horreur, des limites de cette représentation et de sa transcendance, ou de sa non-transcendance, politique, et aussi de la question de savoir si les arts du visuel peuvent construire des "lieux". Si elles peuvent jouer un rôle dans la restitution du disparu au temps historique duquel il a été arraché ${ }^{1}$. » Tomás Ruiz-Rivas

1. Notes inédites de Tomás Ruiz-Rivas préparées à l'occasion de la rencontre «Homo Sacer. La place des disparus dans l'art », Buenos Aires, novembre 2007. 
$\mathrm{C}$ e mot, « disparus ", n’a pas toujours été appliqué à ceux qui, ayant été des opposants de Franco, ou ayant été désignés comme tels, ont été assassinés et enterrés dans des fosses communes pendant la guerre civile espagnole et, ultérieurement, sous le gouvernement franquiste. L'usage du terme est récent et étroitement lié à l'ouverture des fosses communes en Espagne au début des années 2000. Pour beaucoup, dont Tomás Ruiz-Rivas, cet événement marque une rupture :

«Personnellement, je n'avais pas conscience de ce qu'il y avait sous mes pieds, littéralement, avant de lire, pendant l'été 2000, l'information sur la première exhumation réalisée par l'Association pour la récupération de la mémoire historique, ARMH ${ }^{2}$. Une fosse avec treize corps à Priaranza del Bierzo, un petit village de la province de León ${ }^{3}$.»

La plupart des inhumations dans des fosses communes en Espagne ont eu lieu entre 1936 et 1948. Depuis la première exhumation de 2000, cette association a récupéré environ mille corps dans plus de deux cents fosses. Bien qu'il soit extrêmement complexe de donner une évaluation chiffrée, on peut souligner le fait qu'en Andalousie on estime qu'il y aurait environ 53665 fusillés enterrés dans 648 fosses communes ${ }^{4}$.

Sur cet aspect particulier (l'ouverture de ces fosses et, au-delà, sur ce que fut cette modalité singulière de la disparition des personnes sous le franquisme), il existe au moins deux publications, actuellement disponibles en espagnol et en français ${ }^{5}$. Ce dossier ne prétend pas rendre compte de la complexité d'un phénomène que notre revue a abordé à plusieurs reprises, sous diverses perspectives ${ }^{6}$. Il ne s'agit pas non plus de restituer toutes les caractéristiques et les enjeux que les disparitions de personnes ont eus en Espagne. Centrant l'attention sur la vidéo-installation dirigée par Tomás Ruiz-Rivas (sous le pseudonyme de Tom Lavin) et Günter Schwaiger (cinéaste) 7, ce dos-

2. Sur l'ARMH, voir : http://www.memoriahistorica.org/. Il convient de préciser que, suite à la création de cette association, d'autres associations du même type ont vu le jour en Espagne.

3 . Notes inédites de Tomás Ruiz-Rivas préparées à l'occasion de la rencontre «Homo Sacer. La place des disparus dans l'art ", Buenos Aires, novembre 2007.

4. D'après une recherche effectuée par l'Asociación Memoria Histórica y Justicia Andaluza, l'ARMH, le Foro Ciudadano para la Recuperación de la Memoria Histórica. Cette recherche a donné lieu à un document présenté au colloque international sur " Histoire et Mémoire », organisé par la faculté de philosophie et lettres de Grenade (novembre 2007). On peut lire le compte rendu (en espagnol) sur la page suivante (consultée en mai 2008) :

http://www.memoriahistorica.org/modules.php?name=News\&file=article\&sid=539

5. Silva E., Macías S., Las fosas de Franco, Madrid, Edición Temas de hoy, 2003 (Les Fosses du franquisme, Paris, Calmman-Lévy, 2006) ; Martinez-López F., Guerrillero contra Franco. La guerrilla antifranquista de León (1936-1951), León, Instituto leonés de cultura, 2002 (Guérrillero contre Franco. La guérilla antifranquiste du León (1936-1951), Paris, Syllepse, 2000).

6. Sur les disparitions forcées, voir les numéros 13-14, 24-25, 62, 63 et 67 de Cultures $\mathcal{E}$ Conflits.

7. D'origine autrichienne, Günter Schwaiger est réalisateur et metteur en scène. Il vit en Espagne depuis 1991. Nous publions sur le site www.conflits.org un bref entretien avec lui réalisé en 
sier entend rendre compte de cette expérience et de ses propres questionnements ${ }^{8}$.

Ce que la vidéo-installation « Fosse commune » met en scène, c'est d'abord une carte de l'Espagne, dessinée sur le sol, puis recouverte de terre ${ }^{9}$. Cette terre est issue d'une fosse commune, le public est invité à marcher dessus. En parallèle, sur grand écran et avec le son, on peut voir le film de Günter Schwaiger, Santa Cruz, por ejemplo (film consacré à l'ouverture d'une fosse dans le village de Santa Cruz d'où vient précisément la terre utilisée dans cette installation). Sur d'autres chaînes, mais cette fois-ci sans le son, un court documentaire (également réalisé par Günter Schwaiger) est diffusé, il est consacré au processus de ramassage de la terre ayant servi à la fabrication de la carte ; d'autres écrans donnent à voir des photos de disparus réunies par l'ARMH ainsi que les images issues d'une caméra de surveillance (avec laquelle sont filmées les réactions des personnes face à la carte d'Espagne). Ce format admet de légères modifications selon les lieux où le travail est présenté ${ }^{10}$.

Bien que l'installation repose sur ces divers supports, l'entretien que nous avons réalisé à distance avec Tomás Ruiz-Rivas ${ }^{11}$ s'est tout particulièrement intéressé à l'un de ses aspects: la carte dessinée et remplie de la terre d'Espagne.

CEC : J'ai vu des photos de l'installation "Fosse commune ». Dans une de ces photos, quelqu'un (toi ?) est accroupi, avec des gants blancs, en train d'étaler la terre, donnant forme à la carte d'Espagne. Cette image m'as remis en mémoire les vers de Rafael Alberti: "Les terres, les terres, les terres de l'Espagne... les grandes, les désertes, les plaines esseulées 12 ». Encore mainte-

mai 2008, autour de son film Santa Cruz, por ejemplo, réalisé en 2005 en collaboration avec Hermann Peseckas (Beta SP, 65 minutes). Ce film, consacré à l'ouverture d'une fosse commune dans le village de Santa Cruz de Salcedo, lui a valu en 2006 le prix des arts et de la culture de la ville de Salzburg.

8. Cette présentation comporte par ailleurs une série d'annexes. Il s'agit de trois brefs entretiens. Outre celui avec Günter Schwaiger, un autre avec Marie-Paule Jeunehomme (réalisatrice du film Los Nietos, consacré à la quête des petits-enfants des disparus du franquisme) et un dernier avec Monserrat Sans (avocate représentante de l'ARMH devant l'ONU), tous publiés sur le site www.conflits.org

9. Environ $300 \mathrm{~kg}$ de terre ont été utilisés.

10. Le travail "Fosse commune " a été présenté dans les locaux du Ojo Atómico (décembre-janvier 2006), au Centre Cultural Español, Mexique (août-septembre 2006), à Salzburg (septembre-octobre 2006), à l'ECAT, Espacio Contemporáneo Archivo de Toledo (mars 2007). Il a également donné lieu à une conférence à l'Institut Cervantes à Paris (juin 2006) et à une rencontre à Buenos Aires (novembre 2007).

11. Entretien réalisé à distance par Antonia García Castro, en mai 2008 (Madrid/Buenos Aires), d'abord par webcam, puis par courrier électronique, d'où certaines références de la part de Tomás Ruiz-Rivas à ses réponses « écrites ». Nous traduisons de l'espagnol.

12. Rafael Alberti, Galope, extrait. Nous traduisons. 
nant, ces vers occupent pour ainsi dire tout l'espace. Tu m'as dit que la terre utilisée pour cette installation est actuellement dans des sacs, chez toi. Je voudrais que tu me parles de cette terre. Pourquoi la terre? Comment est venue l'idée de la carte d'Espagne?

Tomás Ruiz-Rivas : Effectivement, celui qui est sur le sol en train de dessiner la carte avec la terre, c'est moi. L'idée de travailler avec la terre est antérieure à l'idée de faire une carte. Lorsque je suis revenu d'un séjour de trois ans au Mexique, en 2000, l'ARMH a fait sa première exhumation, qui a eu une importante répercussion dans la presse. Cela m'a beaucoup impressionné. A cette époque, j'étais axé sur une réflexion sur l'effondrement de ma génération, des contradictions de l'art espagnol et des perversions de nos institutions. C'était une période où l'on était en train de construire des musées partout en Espagne, mais sans contenu ni débat. Au cours de cette réflexion, j'ai commencé à comprendre la profondeur des cicatrices laissées par la dictature dans la société espagnole. L'apparition des treize corps de Priaranza m'a mis face à une dimension du franquisme des plus brutales et, en même temps, des plus symboliques. J'ai alors décidé que je ferai quelque chose avec la terre d'une fosse commune, que je ferai marcher le public d'une exposition sur la terre d'une fosse. Mais je ne savais pas comment, jusqu'à ce que je vois le film de Günter Schwaiger, Santa Cruz, por ejemplo ${ }^{13}$. Pendant deux ans, je m'étais demandé ce qu'il fallait faire avec la terre, et soudain, c'est devenu très clair : une carte de l'Espagne. J'unirais alors deux éléments de forte charge symbolique et émotive pour confronter la société espagnole à ses fantômes.

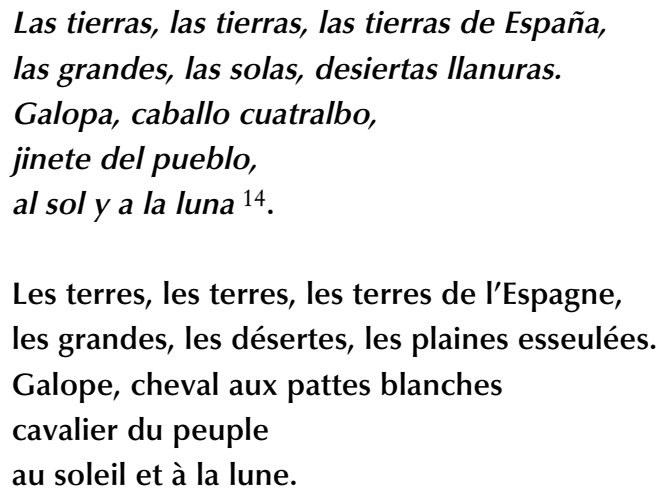

CEC: Tu parles de l'effondrement de ta génération, c'est une expression forte. A quoi fais-tu référence?

13. Voir note 7. Remerciements au Centre culturel espagnol de Buenos Aires qui nous a autorisés à voir le film dans le cadre de la préparation de ce dossier.

14 . Alberti R., Galope, extrait. 
T. R.-R. : C'est un jugement très personnel. J'ai travaillé intensément à Madrid entre 1990 et 1996. Cette période coïncide avec une forte crise économique, avec l'usure du parti socialiste en Espagne et avec l'effondrement du marché de l'art. C'est dans ce contexte que s'est formée, à Madrid, une scène alternative avec une identité générationnelle plus ou moins forte. En 1993 et en 1994, j’ai dirigé le premier Ojo Atómico ${ }^{15}$, j’y ai réalisé un programme d'installations dans un lieu spécifique, c'était une première à Madrid. J'ai également commencé à articuler ma pensée et à acquérir une vision politique de l'art, avec une influence très forte de Manuel Ludeña et de Santiago Sierra ${ }^{16}$. Un de nos sujets de discussion était lié à la nécessité d'interroger les années 1980 (et donc la Movida ${ }^{17}$ ). C'était une nécessité d'ordre générationnel. Il y avait alors deux facteurs importants : le renouvellement des langages, puisque le panorama était dominé par la peinture, et la révision historique avec un fort contenu politique. Lorsque je suis revenu en 2000 , il m'était difficile de comprendre la situation. Les artistes qui avaient renouvelé les langages étaient autres, mais leurs œuvres n'avaient plus aucun contenu politique et ils n'interrogeaient pas les travaux des générations antérieures. Et les artistes avec lesquels j'avais travaillé ne faisaient plus partie de la scène. Tous ces éléments m'ont donné une sensation d'effondrement, et la question la plus évidente " que nous est-il arrivé ? » a rejoint les autres questions que je pouvais me poser à l'époque. J'ai alors compris que, en tant que génération, nous avions un trait commun particulièrement important: nous étions nés dans les années 1960, nous avions grandi sous le franquisme, expérience que la génération suivante n’a pas vécue. Mais cette référence n'a jamais été vraiment présente dans nos esprits. Je crois que ce vide a été déterminant en ce qui concerne la dérive de l'art espagnol vers le non-sens dans lequel il se trouve actuellement.

\section{CEC : Comment se situe " Fosse commune " par rapport à tes autres travaux?}

T. R.-R. : Avant mon séjour au Mexique, je n'étais pas artiste. Je m’explique : bien qu'ayant étudié les beaux-arts, mon champ d'intervention était l'organisation d'expositions de manière indépendante. Concrètement, j'avais eu un rôle plus ou moins important dans les années 1990, en tant que promoteur d'une scène alternative qui n'existait jusqu'alors pas à Madrid. Mon expérience au Mexique et le fait de retrouver la société espagnole m'ont poussé à reprendre la pratique artistique. Ce qui est arrivé, c'est que j'ai évolué d'une analyse ironique, festive on pourrait dire, de l'identité espagnole, vers un travail très politisé sur le fascisme. Pour le moment, je reste centré sur la question de la mémoire. Mon dernier projet, qui n’est pas encore bouclé, est celui du

15. Voir : http://www.ojoatomico.com

16. Selon les mots de Tomás Ruiz-Rivas l'importance du trio composé par ces deux artistes espagnols contemporains et l'artiste allemande Almut Linde est d'avoir « développé une esthétique marxiste, dans un langage très actuel».

17. Mouvement culturel espagnol qui a accompagné le processus d'ouverture politique, suite à la mort de Franco, et plus particulièrement au début des années 1980. 
Musée de la défense de Madrid ${ }^{18}$, et j'en ai d'autres en tête sur la même ligne. Mais je suis également intéressé par la possibilité de travailler sur le franquisme aujourd'hui, sur les traces qu'il a laissées et que l'on peut suivre, pour ne donner qu'un exemple, dans l'urbanisation de la côte méditerranéenne.

\section{CEC : Pourrais-tu me préciser un peu en quoi l'expérience mexicaine à} laquelle tu fais référence a été fondamentale?

T. R.-R. : Le Mexique m'a permis de comprendre l'Espagne, et donc beaucoup d'aspects de moi-même. C'est une société qui a une tradition moderne très importante, depuis l'Indépendance, et qui au XXe siècle a connu une révolution et, de ce fait, une refondation. C'est tout le contraire de l'Espagne, où il n'y a pas eu de modernité, et où notre triste mythe fondateur est le coup d'Etat et la dictature de Franco. De plus, j'ai eu la chance de vivre une période très intense de l'art mexicain, la seconde moitié des années 1990. C’est une génération de rupture, avec beaucoup de zones d'ombre, sans doute, mais qui a été capable de se réinventer elle-même et de réinventer bien des choses au sein de l'imaginaire mexicain. C'est là que j'ai compris qu'en Espagne, du moins à Madrid, il n'y avait pas eu une génération de rupture.

CEC : Bien, pour en revenir à la vidéo-installation "Fosse commune ", quelles ont été tes motivations en engageant ce travail? Quelles étaient les attentes?

T. R.-R. : C'est arrivé en 2005, un an avant le soixante-dixième anniversaire du coup d'Etat du général Franco. Günter [Schwaiger] était en train d'organiser une série d'activités pour ces dates. Il voulait qu'on organise quelque chose à l'Ojo Atómico et je voulais lui proposer une collaboration dans le cadre d'une vidéo-installation, autour de la carte et de son documentaire. Nos motivations étaient similaires. En premier lieu, il s'agissait de dénoncer une situation non résolue, celle des disparus espagnols. Cette dénonciation venait également en appui des membres de l'ARMH, qui avaient réalisé l'exhumation filmée par Günter et qui, depuis 2000, sont en train de mener un dur travail sur cette question. Ils ont exhumé plus de mille corps. Mais, pour Günter, qui est autrichien, la relation avec le franquisme et sa terreur est bien entendu différente. Je crois qu'il y voit la projection du nazisme dans son pays d'adoption. Il vit depuis une quinzaine d'années en Espagne et il dispose de certaines ressources intellectuelles et émotionnelles pour y faire face, puisque très jeune, à l'école primaire, on lui a appris à assumer un passé collectif imprégné par le mal, et à vivre avec cela. Nous, nous ne savons comment faire face à ce passé. Je crois que les secteurs de droite, tout particulièrement, ont un problème plus psychologique que politique, parce qu'ils ne sont pas encore capables d'assi-

18. Voir le document, plus loin dans ce dossier. 
miler le fait que leur grand-père, leur " papy », pourrait être un fasciste assassin. Mais c'est une autre question. Mes motivations sont complexes, parce qu'elles engagent une descente aux enfers de la conscience collective espagnole, qui me paraît nécessaire pour poursuivre mon travail artistique, et une analyse aussi de la capacité politique de l'art, liée aussi bien à la dénonciation qu'à quelque chose de plus subtil, que j'ai compris en voyant les réactions $\mathrm{du}$ public : la « gestion » de la douleur, la capacité d'objectiver la douleur pour la convertir en quelque chose de maniable.

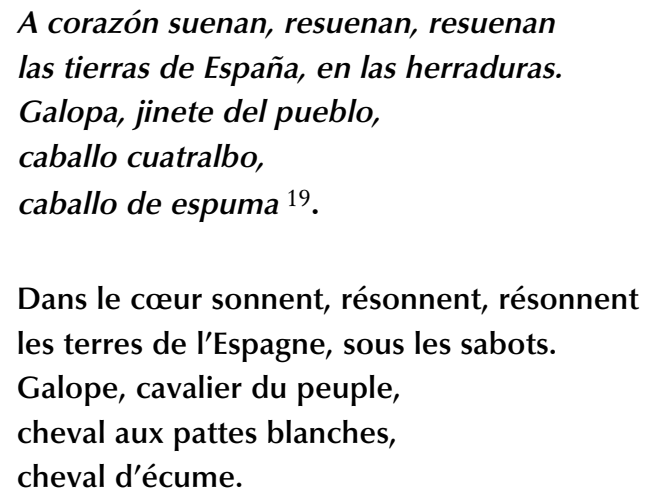

Dans le cœur sonnent, résonnent, résonnent

les terres de l'Espagne, sous les sabots.

Galope, cavalier du peuple, cheval aux pattes blanches, cheval d'écume.

CEC : Concrètement, comment as tu engagé ce travail? Je voudrais pouvoir visualiser la scène. Où as-tu été chercher la terre qui est ensuite étalée pour constituer la carte? Quand, comment et avec qui es-tu allé la chercher?

T. R.-R. : Au cours de la première conversation, Günter et moi nous sommes mis d'accord sur les deux éléments principaux de l'installation : la carte et la projection de son film. La salle du Ojo Atómico faisait environ $120 \mathrm{~m}^{2}$ et il était facile d'imaginer le montage. La carte devait être aussi grande que possible, le sud orienté vers l'entrée de la salle d'exposition, et touchant presque les murs pour que les gens ne puissent pas l'entourer. Et la projection devait se faire sur le mur situé face à l'entrée, de l'autre côté de la carte. Mais, au cours des conversations suivantes, nous avons nuancé beaucoup de détails : pour moi, il était important qu'il y ait un document qui authentifie l'origine de la terre, c'est pour cela que nous avons décidé de filmer le ramassage. Dans la mesure où Günter connaissait déjà les gens de Santa Cruz de la Salceda, il a téléphoné au propriétaire du lieu où se trouvait la fosse pour lui demander la permission, et aussi aux parents proches des six victimes, pour être sûr que le projet ne blesserait pas leur sensibilité. Ils nous ont tous complètement soutenus et l'un des proches, Luis Gonzalo, a apporté ses propres outils et m'a aidé à creuser et à remplir les sacs.

19. Alberti R., Galope, extrait. 
En dehors de ces éléments, nous en avons inclus d'autres dans l'exposition: des textes historiques, comme les déclarations brutales des généraux Mola et Queipo del Llano 20, la déclaration des Nations unies sur les disparitions forcées ${ }^{21}$, à laquelle l'Espagne souscrit mais qu'elle n'applique pas, une liste des exhumations réalisées par l'ARMH, une carte des fosses, etc.

Mon idée était que le public efface partiellement les contours de la carte, pour symboliser cette disparition de la mémoire collective, et j'ai placé tout en haut une caméra de surveillance pour enregistrer l'ensemble du processus. Le fait est que les gens marchaient sur la terre avec beaucoup de respect, ou alors ils ne marchaient pas, ne mettaient pas les pieds dessus; seule la pointe de Tarifa, au sud de l'Espagne a été effacée. Les enregistrements des gens marchant sur la carte sont très bien. A partir de l'exposition à Salzburg, nous avons décidé que je «dessinerai » moi-même la carte, devant les gens, et l'installation a gagné en force.

CEC: A propos de la caméra à laquelle tu fais référence: les gens savaient? Ils avaient conscience d'être filmés?

T. R.-R. : La caméra était connectée à un moniteur où l'on voyait la scène en temps réel. A l'origine, l'idée était que, si la carte devait être entièrement effacée du fait des pas des gens, il y ait une trace. Mais comme les gens essayaient de ne pas trop marcher dessus, le résultat ce sont des images d'un public hésitant, ou entourant la carte sans y marcher, ou y marchant avec un soin extrême. A Tolède, seuls les responsables de la culture du département de Castilla la Mancha ont marché sur la terre, les autres personnes n'ont pas voulu le faire. A Salzburg, en revanche, les gens entraient à l'intérieur de la carte avec beaucoup de respect, conscients de faire quelque chose d'important.

20. «C'est pourquoi je donne pouvoir à tous les citoyens pour que, lorsqu'ils trouveront sur leur chemin l'un de ces individus, ils le fassent taire d'une balle. Ou qu'ils me l'amènent, je la leur mettrai moi-même. Ils connaîtront mon système : pour chaque mort de notre camp, je tuerai au moins dix extrémistes », Proclame du 23 juillet 1936, général Gonzalo Queipo del Llano y Sierra. "Il faudra prendre compte du fait que l'action devra être violente à l'extrême pour réduire l'ennemi aussi vite que possible. Concernant les militaires qui n'auront pas rejoint notre mouvement, les exclure et ne pas les payer. Concernant ceux qui auront pris les armes à notre encontre, contre l'armée, les fusiller. Si je vois mon père dans les files contraires, je le fusille. Quiconque sera ouvertement ou secrètement défenseur du Front populaire devra être fusillé. Il faut semer la terreur, créer la sensation de pouvoir en éliminant sans scrupules et sans hésitation tous ceux qui ne pensent pas comme nous », Instruction réservée n` 1 du 25 mai 1936 signée par «Le Chef» (attribuée au général Emilio Mola Vidal). Ces fragments nous ont été communiqués par Tomás Ruiz-Rivas (nous traduisons), ils sont cités également dans le livre d'Emilio Silva et Santiago Macías, op. cit.

21. Il existe actuellement divers documents internationaux relatifs aux disparitions forcées. Parmi ces documents, on trouve la déclaration de 1992 de l'ONU sur la protection de toutes les personnes contre les disparitions forcées (résolution 46/133) et, beaucoup plus récemment, la Convention internationale pour la protection des personnes contre les disparitions forcées, adoptée le 20 décembre 2006. 
CEC : Revenons sur quelque chose que tu as évoqué brièvement au sujet de la "capacité politique de l'art». Si on pense à d'autres expériences que celle de l'Espagne, un doute persiste : et si la douleur était précisément ce que l'on ne peut jamais "gérer"? Ni à partir de l'art, ni à partir des sciences sociales? Et n'est-ce pas là une impuissance que nous avons en commun, nous qui travaillons -à partir de perspectives, certes, différentes - sur ces questions ou d'autres, similaires, liées aux conflits, pour ne pas dire aux désastres politiques?

T. R.-R. : Maintenant que je lis le mot « gérer » en relation à la douleur, il ne me plaît pas beaucoup. Lorsque je l'ai écrit, je n'ai pas voulu m'arrêter pour penser un terme plus exact. Je crois que quand l'art porte sur la douleur, sur ce que des êtres humains infligent à d'autres êtres humains, et notamment dans le schéma concret des luttes de classes, il a la même capacité et la même impuissance que n’importe quelle autre activité politique ou culturelle. Personnellement, je crois qu'il a, oui, la capacité d'articuler la douleur avec d'autres réalités politiques et historiques, et de lui donner du sens. C'est quelque chose de plus complexe qu'une cérémonie de deuil, dans laquelle l'art peut prendre part de toutes les façons. La manière d'agir de l'art est différente, et ses délais sont différents par rapport à ceux de l'action politique directe. Nous, nous comprenons la réalité à travers des symboles, et dans cette perspective l'art, en tant que producteur de symboles, peut contribuer à changer notre perception des faits douloureux, ou de la douleur elle-même.

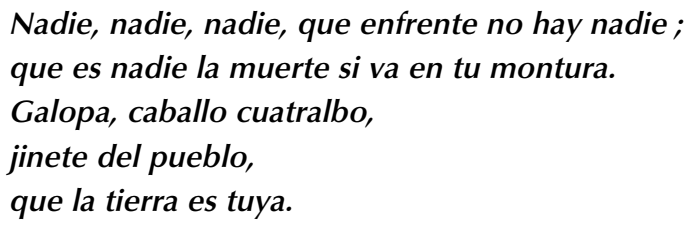

Nadie, nadie, nadie, que enfrente no hay nadie; que es nadie la muerte si va en tu montura.

Galopa, caballo cuatralbo, jinete del pueblo, que la tierra es tuya.

Personne, personne, personne, en face il n'y a personne ; la mort c'est personne si elle va sur ta monture.

Galope, cheval aux pattes blanches,

cavalier du peuple

car la terre est à toi.

CEC : Comment vit-on avec ces terres d'Espagne... dans sa propre maison?

T. R.-R : Les sacs sont au sous-sol, dans un petit débarras, ils ne sont pas dans mon salon. Mais on prend des distances avec les contenus à force de travailler sur ce matériel. Je dois dire que, en général, le travail sur les disparus me provoque une forte angoisse, mais non la présence des sacs, sinon la préparation des expositions, les recherches historiques... Quand tu lis les témoignages, ça te glace le sang. 
CEC : J'ai lu le livre d'Emilio Silva et de Santiago Macias, et, effectivement, lorsque je lis les témoignages, "ça me glace le sang ". La sensation est celle d'un accablement total. L'Espagne semble une vaste fosse commune. Il y a quelque chose qui m'impressionne beaucoup, quelque chose qui distingue l'histoire espagnole de l'histoire chilienne et argentine, par exemple. C'est la grande, l'énorme quantité de témoins qu'il y a eu au moment des faits. Si j'ai bien lu: ceux qui ont assassiné et fait disparaître en Espagne n'ont pas particulièrement cherché à cacher leurs actes. Je comprends que ton travail participe d'un processus de focalisation sur quelque chose qui n'était pas totalement inconnu, mais de quelque chose qui n'a pas été public, officiel, reconnu, assumé comme vérité irréfutable de la guerre civile espagnole et de ses suites. Ton travail, celui de Günter, et aussi - mais autrement - celui de l'ARMH, semble assurer un relais aux mémoires dispersées des familles et des témoins qui n'ont pas soutenu Franco, qui ont été des opposants de Franco. Le changement radical (à mes yeux, tu me diras si je me trompe), c'est la possibilité, aujourd'bui, d'entreprendre des actions conjointes, coordonnées, pour que ces souvenirs, ne périssent pas en même temps que les témoins directs. Ça a un sens pour toi de poser la question en ces termes? Ou est-ce que je passe à côté d'une autre dimension qui, pour toi, serait fondamentale?

T. R.-R. : Les responsables ne cachaient pas les massacres, en effet, parce que l'objectif était de semer la terreur. Un des motifs de la défense acharnée de Madrid 22 a été que les réfugiés qui venaient d'Andalousie et d'Extremadour racontaient de telles horreurs que les gens préféraient mourir plutôt que d'être arrêtés. Je ne sais plus si dans le livre d'Emilio on raconte le procédé qui a été adopté dans toute cette zone (Castilla-León), où il n’y a pas eu de front de guerre : les fascistes (et le curé) de chaque village faisaient une liste avec les gens qu'il fallait tuer, puis ils l'échangeaient avec les listes élaborées dans d'autres villages; ainsi ils n'étaient pas contraints de tuer des gens qu'ils connaissaient ; j'ignore s'ils y auraient vraiment vu des inconvénients... Mais, dans le cas où la zone aurait été prise par les républicains, il n'aurait pas été si facile de désigner les coupables ${ }^{23}$. Bref, c'est un autre problème. En ce qui concerne ta question, je crois que le travail des associations pour la mémoire historique, les forums pour la mémoire et celui des artistes et des intellectuels qui s'occupent de ces questions est très important pour plusieurs raisons : d'abord, pour tenter d'en finir avec la négation de l'évidence; mais aussi pour construire une autre image de nous-mêmes. En tant que société, en tant que pays, venir à bout d'un traumatisme collectif qui n'est même pas assumé comme tel $[\ldots]$.

22. Voir plus loin dans le dossier le document consacré au « Musée de la défense de Madrid».

23. Ainsi, même si les assassinats étaient souvent perpétrés devant témoins et que les villageois pouvaient savoir où se trouvaient les fosses communes, il y a bien eu, parfois, processus de dissimulation, concernant un aspect précis : l'identité des coupables. 
Pour moi, à partir de l'art, il est très important de mettre en question un certain état des choses donné pour sûr. J'en suis venu à cette ligne de travail en essayant de comprendre pourquoi l'art espagnol est si banal, et ce que j'ai découvert c'est une série de prémisses que le monde de l'art a acceptées inconsciemment. C'est un vaste thème, mais il met en évidence de quelle manière les structures de la connaissance, le système vertical des relations sociales, le caractère obligatoire de l'éducation catholique, etc. conditionnent très profondément la pensée espagnole. Il y a quelques textes sur cela, mais ces thèmes sont traités de façon insuffisante. Je pourrais dire, si tu me permets d'adopter un ton plus littéraire, que sans le savoir, chaque Espagnol a une fosse dans son inconscient, et que pour avancer, en tant qu'intellectuel ou en tant qu'artiste, mais aussi en tant que sujet politique, il doit la trouver et l'ouvrir. 


\section{DOCUMENT \\ Manifeste du Ojo atómico. Antimuseo de arte contemporáneo (extraits) 24}

L'être humain pense avec des images. Qu'est-ce que cela veut dire ? Que nous sommes capables de former dans notre esprit des représentations de choses qui existent ou qui n'existent pas, de donner à ces représentations des valeurs symboliques et de les exprimer sur un support donné, en vue de les partager. C'est le principe commun et lointain des arts du visuel dans toutes les cultures, et il se fonde sur la nature même de l'être humain. [...]

L'Antimusée est un centre expérimental d'art contemporain. Nous considérons que les arts du visuel peuvent et doivent être un instrument d'émancipation. Que dans un panorama dans lequel les pratiques politiques traditionnelles ont perdu du sens, la subversion culturelle est l'un des champs d'action les plus féconds pour générer des espaces de résistance, fortifier la démocratie et préserver la diversité sociale.

Notre objectif est de développer de nouvelles formes de publicité et de relation avec la société. Contrairement aux modes de visibilité caractéristiques de l'industrie culturelle - présence dans les médias et célébrité - l'Antimusée travaille sur la construction des publics. Il encourage des communautés de communication éphémères, mais capables de produire du sens et d'articuler entre elles différentes sphères contre-publiques [...].

\section{Présentation du projet La Defensa de Madrid 25}

A partir de la vidéo-installation «Fosse commune », exposée pour la première fois en 2005 dans l'Antimusée, mon travail s'est complètement centré sur la recherche autour du franquisme et ses conséquences, sous tous les aspects et dans tous les milieux imaginables [...]. Le Musée de la défense de Madrid prend place dans ce même processus de travail et il se rattache, à l'origine, à une autre présence souterraine : celle d'un refuge anti-aérien situé dans les sous-sols de la rue Juan Bautista de Toledo, très près du siège actuel de l'Ojo Atómico. Le quartier de Prosperidad était dans les années 1930 un lieu périphérique, non relié par le métro (dont les tunnels ont été, ailleurs dans le centre-ville, utilisés comme abris lors des attaques de la Luftwaffe nazie). Ce refuge est fermé depuis près de soixante-dix ans et presque tout le monde l'a oublié.

Ma première idée était de travailler sur cet emplacement même, profitant du potentiel symbolique que suppose sa simple existence cachée. Il s’agissait

24. Le texte complet est disponible sur le site : http://www.ojoatomico.com

25. Extraits de l'article «Museo de la Defensa de Madrid ", publié sous le pseudonyme de Tom Lavin dans Ramona, n78, mars 2008, Buenos Aires, pp. 37-41. 
de récupérer sa mémoire - à travers des témoignages de personnes âgées et par le biais de certaines actions -, en tant que symbole de la lutte hérö̈que que le peuple de Madrid a mené contre le fascisme (lutte condamnée à l'oubli suite à une série de moments ou d'épisodes historiques qui ont fini par légitimer le franquisme : en particulier le pacte de Madrid de 195326 et la transition des années 1970).

Mais j'ai rapidement compris que la défense de Madrid était un fait historique qui dépassait largement ce que je pouvais faire à partir du refuge. En résumant beaucoup, on peut dire que Madrid était en 1936 une ville indéfendable militairement, avec un gouvernement qui avait perdu le contrôle de la situation et qui s'était déplacé à Valence, sans une véritable armée qui puisse faire face aux troupes soulevées [...].

Mais le fait est que Madrid a résisté. La ville a résisté au premier assaut, en novembre 1936, et pendant toute l'année 1937, et pendant toute l'année 1938, jusqu'à la chute en mars 1939. Il y a eu des volontaires du reste de l'Espagne, les membres des brigades internationales et des intellectuels antifascistes comme Hemingway et Malraux, sans parler de tous les écrivains et artistes espagnols qui ont décidé de rester dans la ville jusqu’à la fin. Madrid est devenue le symbole international de la lutte contre le fascisme, dans ces années obscures de la montée du national-socialisme, avant la Seconde Guerre mondiale.

Dans le contexte politique de ces deux dernières années de grande crispation et avec un débat vraiment animé à propos de la loi de la Mémoire historique ${ }^{27}$, l'idée de mettre en place un musée de la défense de Madrid a pris pour moi un caractère d'évidence. En Espagne, il n'y a pas de musées consacrés à la guerre civile (exception faite d'un musée privé, qu'un monsieur a improvisé dans le grenier de sa maison dans un petit village appelé Mohedas de la Jara, à Tolède ; exception faite aussi des mémoriaux franquistes et/ou catholiques, comme par exemple, el Valle de los Caídos ${ }^{28}$ ). [...] Je signale que la mairie m’a systématiquement refusé toutes les autorisations nécessaires pour montrer le musée ${ }^{29}$ dans des espaces publics, ce qui ne m'a pas empêché de le montrer quand même, bien sûr.

26. Le pacte de Madrid comprend concrètement trois accords bilatéraux entre les Etats-Unis et l'Espagne dans les domaines de défense, d'économie et de technique. Sa signature constitue en soi une légitimation du gouvernement franquiste.

27 . Adoptée le 31 octobre 2007 par la Chambre des députés, elle prévoit entre autres la reconnaissance des victimes de la guerre civile (des deux camps), des victimes de la dictature, l'ouverture des fosses communes.

28. Littéralement "Vallée de ceux qui sont tombés». Monument commandé par le général Franco pour rendre hommage aux combattants franquistes morts pendant la guerre d'Espagne.

29. Comme Tomas Ruiz-Rivas l'explique ci-après, le musée est itinérant, d'où l'expression «montrer». 
J'ai considéré plusieurs idées pour le musée. [...] Finalement, je me suis décidé pour un musée ambulant. Un objet de nature sculpturale, mais qui s'active seulement comme performance et que les gens peuvent interpréter aussi bien dans sa dimension artistique que politique. J'assumais la précarité de la mémoire historique espagnole par le biais d'un objet difficile à classer. [Au cours de ce travail], j'avais découvert que la société, et la vie telle qu'elle s'est déroulée dans la ville assiégée, avec les bombes, la peur et la faim, mais aussi avec la décision inébranlable de résister, ont laissé une infinité de petites marques que les quarante années de dictature n'ont pas réussi à effacer. Un musée ambulant pouvait dévoiler in situ ces marques et re-signifier l'espace urbain [...].

Le Musée de la défense de Madrid est partial : il ne prétend pas porter un regard scientifique et supposé impartial sur le passé, mais récupérer et rendre hommage à la mémoire de ceux qui ont lutté pour la liberté. [...]

Par ailleurs, j’ai décidé de ne pas y inclure des symboles ayant une actualité politique, tels que le drapeau républicain ${ }^{30}$, la faucille et le marteau ou les sigles des différents partis et syndicats qui ont pris une part active à la défense de la ville, parce qu'il m'a semblé qu'à travers ces symboles, s'établiraient des liens trop directs avec des questions politiques actuelles, et que cela limiterait l'ampleur des registres que permet ce travail.

Je n'ai pas prévu de date de fin pour ce projet. Le musée est actif, je continuerai à augmenter ses fonds documentaires et à le sortir dans la rue indéfiniment.

30. Au sujet de cette question et notamment de l'usage des drapeaux, voir également en annexe (sur le site de la revue) l'entretien avec Günter Schwaiger. 